\title{
Whatsapp y Facebook: Análisis del uso de herramientas síncronas para la comunicación en la educación unbiversitaria
}

\section{Whatsapp and Facebook: Analysis of the use of synchronous tools for communication in university education}

\author{
Mtro. Luis Enrique Garcia Alvarez ${ }^{1}$ \\ Universidad de Guadalajara \\ Centro Universitario del Norte \\ Dr. Martín Eliseo Tamayo Ancona ${ }^{2}$ \\ Universidad Internacional Iberoamericana
}

\section{RESUMEN}

La manera en la que nos comunicamos ha cambiado de forma importante. Ejemplo de ello es el uso recurrente que los alumnos hacen de los dispositivos móviles, por ejemplo, los conocidos comúnmente como Smart Phone. El uso de dispositivos móviles permite métodos de comunicación cercanas a los estudiantes, por lo que se optó por herramientas tales como

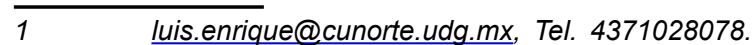
Colotlán, Jalisco, México. Académico del Centro Universitario de Norte (UDG). Estudiante del Doctorado en Educación en la Universidad Internacional Iberoamericana. ORCID: https://orcid.org/0000-0002-6194-3577

2 martin.ancona@unini.edu.mx, Tel.9811037851. Campeche, Campeche, México. Docente del Doctorado en Educación en la Universidad Internacional Iberoamericana. ORCID: https://orcid.org/0000-0003-1020-1300
WhatsApp y Facebook.

Al utilizar estos recursos de mensajería instantánea surge la interrogante: ¿En qué medida los alumnos expresan su satisfacción en la comunicación en la educación semipresencial en CUNorte, con la incorporación de herramientas síncronas en línea? Para lograr dar respuesta a dicha interrogante se han contrastado las respuestas en 2 grupos del tercer semestre de la Ingeniería en Electrónica y Computación (IEC), asignados en el ciclo 2019B (julio-diciembre). El Grupo " $A$ " ha utilizado WhatsApp y Facebook para su comunicación, el Grupo "B" únicamente utilizó Moodle. Lo anterior se llevó a cabo en el Centro Universitario 
del Norte (CUNorte) de la Universidad de Guadalajara (UDG) en un ambiente de tipo B-Learning.

\section{PALABRAS CLAVE}

Educación, WhatsApp, Facebook, Comunicación, TIC.

\section{ABSTRACT}

The way we communicate has changed significantly. An example of this is the recurrent use of mobile devices by students, for example, those commonly known as Smart Phones. The use of mobile devices allows communication methods close to students, so tools such as WhatsApp and Facebook were chosen.

When using these instant messaging resources, the question arises: To what extent do students express their satisfaction with communication in blended learning at CUNorte, with the incorporation of synchronous online tools? In order to answer this question, the results of two groups from the third semester of Electronic and Computer Engineering ( $\mathrm{ECl})$, assigned in the 2019B cycle (July-December), have been compared. Group "A" has used WhatsApp and Facebook for its communication, Group "B" only used Moodle. The above was conducted in the Centro Universitario del Norte (CUNorte) of the University of Guadalajara (UDG) in a B-Learning type environment.

\section{KEYWORDS}

Education, WhatsApp, Facebook, Communication, ICT.

\section{INTRODUCCIÓN}

La investigación surge por el interés de realizar un análisis de las herramientas educativas que se utilizan tradicionalmente en la Educación a Distancia. La comunicación en la educación es el eje principal a través del que se integran los grupos intelectuales, volviéndose una necesidad básica entre docente y alumnos (Vaca el al., 2016). Al no lograr una comunicación fluida y constante se pierde el sentido del aprendizaje (Marrero, 2016). Es de notar que al usar una única herramienta para educar y comunicar (Moodle), se evita un análisis temprano de instrucciones que pudieran ser confusas al estudiante y se limita el consenso general sobre los productos a desarrollar.

Se entiende a la educación como uno de los ejes centrales de la universidad ya que debe concentrar sus esfuerzos en preparar al profesionista que hará frente a los problemas que aquejan a la sociedad; esta tarea tan importante se vuelve una búsqueda constante de mejora en sus procesos educativos y es donde como profesores de la misma, se debe ser partícipe activo del desarrollo de propuestas educativas.

La investigación desarrollada permitió realizar un análisis sobre la satisfacción del alumno con respecto a la comunicación al utilizar herramientas asíncronas, como Moodle, para comparar los datos con un grupo que hace uso recurrente de WhatsApp y Facebook, de tal forma que se determine la estrategia con la que los alumnos se sientan más identificados y se promueva una comunicación que se desarrolle de alumno-profesor y alumno-alumno.

El nivel en la calidad educativa en las instituciones que brindan algún grado de preparación a través de las tecnologías de la información como medio de soporte, conocidos como: a distancia, semipresencial, en línea, B-Learning, E-Learning, M-Learning, etc., es cuestionado, muchas de las veces por los mismos alumnos que cursan dichas modalidades educativas, sobre todo por no sentir que existe un docente que dé respuesta de forma rápida a sus inquietudes o simplemente al no hacerlo. Santoveña (2012), indica cuatro desventajas de utilizar herramientas asíncronas las cuales son: retraso en la respuesta/falta de respuesta inmediata, 
el desarrollo del sentimiento de aislamiento, la falta de tiempo para la discusión y la interacción.

Solana (2011), indica "más del $50 \%$ de la mejora educativa es resultado de la intervención exitosa del instructor, de su habilidad para enseñar, de su profesionalismo y dominio de las habilidades y conocimientos que habrá de impartir." ( $p$. 20), creemos que a partir de utilizar medios de comunicación con los que el alumno se identifica, dicho porcentaje se pueda incrementar y se logre que el aprendizaje de los alumno sea aún mayor.

\section{SUSTENTACIÓN}

\section{SUSTENTACIÓN TEÓRICA}

Es factible identificar a la universidad como ente guiador de sociedades, desde ella es posible la trasformación del pueblo, pues es en ella donde se posibilita la convivencia de diferentes mentalidades y estados culturales, al existir diversidad de pensamientos se origina la diversidad de soluciones.

El sistema educativo nacional mexicano es regido por la Secretaria de Educación Pública (SEP), dicha institución ha definido las modalidades bajo las cuales una institución educativa puede ofrecer programas de formación, según el artículo número 46 de la Ley General de Educación, la educación en México posee tres modalidades: escolarizada, no escolarizada y mixta (Secretaria de Educación Pública, 1993).

Resulta importante observar que dentro de la educación en línea existen variedades y no se trata de un proceso unificado, Cook (2013) indica la necesidad de observar la existencia de múltiples formas de aprendizaje en línea al igual que lo existen de forma presencial; por ejemplo, E-Learning, B-Learning, M-Learning, por mencionar algunos, en nuestro caso y para nuestro estudio nos hemos enfocado en la modalidad B-Learning.
¿Pero cómo es que surge el sistema conocido como $B$-Learning? El primer término que surge de la incorporación de la educación a distancia con el uso de las tecnologías de la información y comunicación (TIC), es conocido como E-learning así lo expresa Turpo (2013), el mismo autor indica que el termino Blended Learning, también conocido como B-Learning, surge de realizar un híbrido en la educación, es decir, la educación semi-presencial es sustentada con las TIC.

Padilla (2008), ha definido la educación a distancia en 2 tipos: "sincrónico y asincrónico", el primer caso es identificado de forma similar al proceso educativo en el aula con sesiones cara a cara, en el segundo caso se vierten los contenidos a través de plataformas para que el alumno los consulte cuando él lo considere adecuado.

Existen cuatro elementos muy importantes en la educación identificados por George y Trujillo (2018), dichos elementos son: el profesor, los estudiantes, el contenido y la tecnología que se usa para la enseñanza y el aprendizaje, los autores resaltan que de la interacción entre los cuatro factores se posibilita logra impactar en el aprendizaje. El uso de tecnologías en la educación ya no es una novedad, ni siquiera es una acción innovadora, se ha vuelto una actividad cotidiana que día a día se incorpora a las universidades así lo expresa Hernández (2017).

Utilizar dispositivos y/o herramientas de uso cotidiano por el alumno se ha vuelto de suma importancia, ejemplo de ello es el celular, en este sentido Cisneros y Robles (2017), han encontrado que es utilizado para buscar información, comunicarse a través de diferentes aplicaciones y formar grupos sociales, otros datos importantes al respecto indican que para el 2012 el crecimiento de dispositivos celulares fue de un $707.41 \%$, y para el 2013 se reportó que el $96.1 \%$ de los hogares tienen teléfono 
móvil según Herrera et al., (2017), un ejemplo más cercano es el caso CUNorte, ya que la Coordinación de Tecnologías para el Aprendizaje indicó en el 2017 que el 70\% de los dispositivos para los que se demanda acceso a internet son celulares, estos datos reflejan el fuerte aliado que puede ser un celular en la educación.

Con el uso de aplicaciones es posible hacer partícipes a los alumnos y sus compañeros en la retroalimentación, al utilizar herramientas sociales como es el caso de Facebook, los alumnos crean grupos que son utilizados para desarrollar sus actividades académicas en conjunto según Cisneros y Robles (2017). Los tiempos de respuestas se acortan considerablemente, los alumnos son capaces de establecer comunicación constante con sus compañeros y su profesor, basta con utilizar una de las aplicaciones que ofrece el celular o bien realizar una llamada, así lo expresan Alonso et al., (2016).

El proceso educativo se sustenta en indicaciones claras y nutridas, al respecto Mora (2014), indica: los cursos en línea requieren comunicación clara por parte del tutor, lo cual no siempre es sencillo cambiando conforme a la variedad de herramientas en las plataformas y la interacción a través de ellas. WhatsApp es una aplicación idónea para que el profesor pueda realizar retroalimentación de las interrogantes de sus alumnos e incluso desarrollar evaluaciones diagnósticas según lo expresado por Sanz (2014), de la misma forma el autor cita a Hidalgo (2013), e indica que los alumnos ya hacen uso de la herramienta para la educación sin que ningún profesor lo haya propuesto.

El uso de herramientas de comunicación como lo es WhatsApp, que además incorporan toda una gama de contenidos multimedia (audio, video, imágenes, documentos, etc.) que pueden ser utilizados para el aprendizaje, ya han sido puestas a prueba en diferentes instituciones educativas, por ejemplo, los estudiantes de
Odontología de Sao Paulo, Brasil, en dicha investigación los autores Giasanti et al., (2016) identifican: WhatsApp es una aplicación que es parte de la rutina de las personas, ya que los aparatos tienen precio de mercado más accesible, los autores han citado a Ibrahin, Hafis e Idri (2015) e identifican que WhatsApp puede ser la mejor ayuda para alumnos introvertidos, ya que hay una ausencia o disminución de la ansiedad, evitando la necesidad de cuestionamientos a profesores y colegas en la clase.

Por otro lado, es el profesor quien conoce de primera mano las necesidades propias de sus estudiantes en el proceso de innovación educativa. Al respecto Alfaro et al., (2014), identifican al profesor como pieza fundamental en la innovación educativa ya que son los ejecutores de la propuesta de aprendizaje al conocer mejor las necesidades y problemáticas específicas de los alumnos, de la misma forma indican la existencia de formación pero poco cambio, la formación no es proporcional a la innovación que la sociedad exige, para ello proponen potenciar una cultura formativa que genere nuevos procesos de formación y que además de ello se introduzcan nuevas perspectivas y metodologías que se adapten a las necesidades de las personas que aprenden.

El rol que juega el profesor a lo largo de la historia ha sido modificado conforme a los cambios y crisis en la relación sociedadeducación-cultura, el profesor se ha tenido que adaptar a los continuos cambios sociales y avances tecnológicos, según lo expresado por Camacho y Becerra (2016), en el mismo sentido se expresan Fernández et al., (2017), al indicar "por lo dinámico que es el ambiente educativo, el docente deberá adaptarse a los cambios, no solo eso sino emplear los nuevos recursos en su práctica de manera que los estudiantes adquieran conocimientos de calidad y vigentes" (p. 12). 
Dentro de la preparación que un docente debe poseer se encuentra la habilidad de responder a las necesidades individuales, grupales e institucionales que la comunidad educativa presentan, así lo expresan Ruiz et al., (2016).

¿Cómo se define o identifica un buen profesor? La respuesta para esta interrogante podría resultar variada y compleja, e incluso contener varios parámetros, sin embargo Ochoa y Domínguez (2007), citados por Cortez et al., (2013), identifican a un buen docente en base a: dominio de contenidos, habilidades pedagógicas y se comunica de forma eficiente. En base a esto es posible observar que la comunicación es parte fundamental en un docente.

Dentro de las competencias que como docente es necesario poseer se encuentran las competencias digitales, basta con observar las estrategias de educación que se nos plantean y sin lugar a duda parte fundamental son las TIC. Viñals y Cuenca (2016) han identificado 5 dimensiones que se deben cumplir para ser competente digital, las cuales son: 1) información, ya sea para identificarla, recuperarla, almacenarla, organizarla y analizarla, 2) comunicación: compartir recursos, colaborar e interactuar, 3) creación de contenidos, lo cual incluye editar los ya existentes y proporcionar nuevos, 4) seguridad: protección personal, protección de datos y sostenibilidad y 5) resolución de problemas: necesidades, elección de herramientas digitales, resolver problemas conceptuales, resolver problemas técnicos, uso creativo de la tecnología y la actualización de las competencias propias y la de los otros.

Si se quiere alcanzar el aprendizaje del alumno debe ser a través de la comunicación de esta forma lo expresan Hershkovizt y ForkoshBaruch (2017). Para Gómez et al., (2017) es el profesor quien orienta el conocimiento en el alumno, tomando un papel trascendente cuando el alumno no logra comprender y es el docente quien toma las dudas y las vuelve certidumbre.
Según Castro y Jiménez (2008), la comunicación se establece de forma vertical u horizontal, la comunicación vertical es aquella que se genera desde el profesor y desciende al estudiante y la horizontal se genera en los mismos niveles jerárquicos; por ejemplo, entre alumnos.

La comunicación entre alumnos es muy importante como lo indican Medina et al., (2017), al respecto expresan: "un caso particular de la comunicación interpersonal lo constituye, la comunicación inter-alumnos, componente importante dentro la comunicación pedagógica y que ha sido muy poco trabajado con anterioridad" (p. 169). Escobar (2015), indica "La interacción alumno-docente, no sólo, se da entre un alumno y el docente, sino que involucra a todos los alumnos que el profesor tiene en su grupo de clases" (p. 2), además Sepúlveda (2016) identifica a la educación y a la comunicación como procesos innatos ya que surgen de la incorporación de los hombres en un ambiente social.

Una de las frases que más se adentra en nuestra investigación es la expresada por Amayuela (2017) en la cual indica "educación y comunicación son procesos inseparables, ya que cualquier hecho educativo requiere mediaciones comunicativas y no hay situación comunicativa que no tenga una influencia educativa, en algún sentido" (p. 11); es por ello que la comunicación se vuelve inseparable del proceso educativo.

La innovación educativa no puede suceder sin que el docente sea agente de cambio, es decir, si se desea innovar en un contexto educativo la base para ello es el profesor ya que es él quien convive de forma cotidiana con los alumnos y por lo tanto es el promotor ideal en los contextos educativos, ejemplo de ello se expresa en el siguiente apartado: "El plan de comunicación del centro nos va a ayudar difundir la innovación de manera estratégica, seleccionando medios y utilizando motivación, 
liderazgo, clima positivo y el profesor como agente de cambio" (Morales, 2011, citado por Hernández, 2015, p. 3).

Es posible definir a la satisfacción como la "relación con el bienestar personal o el bienestar subjetivo, analizado por la psicología a partir de juicios cognitivos y respuestas emocionales" (Gutiérrez y Gonçalves, 2013, citados por Ramírez e Hidalgo, 2018. p. 3). Álvarez et al., (2015) han encontrado que en primer lugar el alumno valora la actitud del profesor, el desenvolvimiento del docente en la asignatura y factores como la evaluación de los exámenes; en nuestra investigación se resalta la satisfacción del alumno con respecto a la actitud del profesor y del desenvolvimiento del mismo.

Medir la calidad educativa es una de las nuevas estrategias bajo las cuales las universidades han enfocado nuevos esfuerzos, incluso sometiéndose al escrutinio de entes evaluadores, actividad que desde nuestra perspectiva es adecuada, sin embargo, los esfuerzos no deben ser detenidos ahí, al respecto Surdez et al., (2018), identifican la necesidad de interrogar al estudiante ya que es él quien recibe la educación y por lo tanto las consecuencias de la calidad educativa; no podría existir mejor juez que el alumno.

¿Pero que representa la satisfacción en un estudiante o cuál es el carácter de su medición? Al respecto Álvarez et al., (2015) indican: la valoración de la satisfacción de un estudiante determina la calidad educativa, ya que es punto fundamental a través de la cual se mide la eficiencia académica y administrativa, de la misma forma que con las unidades de aprendizaje.

Es elocuente interrogar ¿por qué es necesaria la satisfacción del alumno? En este sentido Ramírez e Hidalgo (2018) expresan la necesidad de relacionarlo con el rendimiento académico. Lo cual nos muestra que un alumno satisfecho es un alumno con un mejor rendimiento académico, que al final del camino es el objetivo que se desea alcanzar, preparar alumnos competentes.

Medir la satisfacción del alumno permite realizar consideraciones orientadas a la toma de decisiones y acciones que motiven la preparación del alumno, al respecto Pérez et al., (2015) han indicado la necesidad de realizar estudios de satisfacción porque inciden directamente en la disminución de las tasas de fracaso y deserción, además de proporcionar información importante para la mejora de la actividad docente.

\section{SUSTENTACIÓN METODOLÓGICA}

La investigación se basa en el paradigma cuantitativo, con enfoque positivista, ya que pretende estudiar un fenómeno situado en la realidad que "está dada y que puede ser conocida de manera absoluta por el sujeto cognoscente" (Dobles y Col., 1998, citados por Pérez y Pereyra, 2015, p. 72), el estudio es de carácter novedoso en la Universidad de Guadalajara, concretamente en el Centro Universitario del Norte, ya que no se ha encontrado ninguna de tipo similar.

La observación desarrolla un análisis del efecto, medido como nivel de satisfacción, que causa en los alumnos la incorporación de algunas herramientas síncrona para la comunicación, dicho enfoque es conocida como causa-efecto o explicativa (López y Fachelli, 2015), ya que a través de la intervención en la variable comunicación (con herramientas síncronas o en tiempo real) se busca medir el estado emocional de los alumno; es preciso indicar que la investigación ha sido desarrollada como cuasi-experimental, ya que fue aplicada con dos grupos (en principio con 20 alumnos cada uno), en la Ingeniería en Electrónica y Computación. En un grupo se emplearon Facebook (mensajero y red social) y WhatsApp como medios de comunicación que complementan a Moodle, en el segundo grupo no se incluyen 
ni Facebook ni WhatsApp, el alumno pudo realizar la comunicación a través de Moodle, correo electrónico o cualquier otro que eligiera, sin realizar comunicación síncrona o en tiempo real por lo que siempre se mantuvo un contacto asíncrono.

Las características con las que cumplen los grupos seleccionados son: cursan el mismo plan de estudio, cursan la misma asignatura, se encuentran en similar semestre, de esta forma se interviene con alumnos donde las condiciones educativas son las mismas, y por lo tanto, es posible identificar de forma más cercana el impacto que conlleva el cambiar las variables comunicativas, la muestra seleccionada no es aleatoria, también conocida por Otzen y Manterola (2017), como no probabilísticas.

En el desarrollo de la investigación cuantitativa, el instrumento de aplicación y/o medición toma gran relevancia, es por ello que se ha optado por el desarrollo de un cuestionario, ya que este es capaz de brindar los datos con los que se fundamenta la investigación la encuesta fue valorada por un grupo de cinco expertos, los cinco han laborado y/o laboran en ambientes E-Learning y B-Learning.

Se han generado dos cuestionarios en el primero se incluyen las dimensiones: Datos genéricos del alumno, Satisfacción del alumno en relación a la comunicación que se tiene entre sus compañeros y su profesor, Satisfacción del alumno como miembro activo de la comunicación, al usar herramientas en línea y finalmente la Satisfacción del alumno con respecto a la incorporación de herramientas síncronas en su educación, con un total de 32 ítems; en el segundo cuestionario se omite la última dimensión indicada, esto es debido a que es aplicable a los alumnos donde no se incluye ningún tipo de herramienta síncrona o de comunicación instantánea, en él se identifican 24 ítems.-
El cuestionario fue aplicado finalmente con 27 alumnos de los cuales 13 forman parte del grupo que utilizó WhatsApp y Facebook como herramientas de comunicación y 14 donde se han omitido dichas herramientas, es evidente que el número de 40 alumnos con el que originalmente se inició el proceso descendió en buena medida por la deserción, lo cual es indicador de la necesidad de realizar un análisis en dicho sentido pero que escapa a los alcances del presente. A excepción de la dimensión Datos personales, cada ítem contiene las escalas: Muy Satisfecho(a), Satisfecho(a), Indiferente, Poco Satisfecho(a) e Insatisfecho(a).

Para el análisis se decidió utilizar la prueba $T$ de Student ya que esta nos permite comparar dos grupos de estudio donde se han sometido a condiciones diferentes de análisis, a este tipo de pruebas son llamadas por González et al., (2017. p. 186) como: Prueba $T$ de Student para muestras Independientes.

Para verificar la normalización de datos se aplicó la prueba Shapiro-Wilks, usando un complemento de Excel llamado Real Statistics, dicho complemento nos permite realizar algunos procedimientos estadísticos de que origen no se encuentran incorporados en el procesador, al realizar el análisis se obtuvieron datos como la prueba $P$-Value, en la cual los resultados para la dimensión Satisfacción del alumno en relación a la comunicación que se tiene entre sus compañeros y su profesor fueron: 0.90997 (Grupo A) y 0.32762 (Grupo B). En el caso de la dimensión el Satisfacción del alumno como miembro activo de la comunicación, al usar herramientas en línea los datos arrojados son 0.16579 y 0.05258 para los mismos grupos de estudio en la prueba $P$-Value.

Nuestro nivel de error permitido es de $5 \%$, es decir, el nivel de significancia es de 0.05 , según Martínez et al., (2016) y Arteaga et al., (2018). Finalmente, con los datos obtenidos se procede a realizar las pruebas $T$ de Student, para dicho 
propósito se utiliza el software PSPP (Free Software Foundation, Inc., 2020), los resultados arrojados por PSPP son presentados en las Tablas 1 y 2 :

Tabla 1. Estadística de grupo.

\begin{tabular}{cccccc}
\hline & Grupo & N & Media & Desviación Estándar & Err. Est. Media \\
\hline \multirow{2}{*}{ Puntaje } & Grupo “A" & 13 & 52.31 & 4.52 & 1.25 \\
\cline { 2 - 6 } & Grupo "B" & 14 & 36.93 & 12.63 & 3.37 \\
\hline
\end{tabular}

La Tabla 1 nos indica: se ha aplicado la prueba a 13 alumnos del Grupo " $A$ ", en dicho grupo se han utilizado WhatsApp y Facebook, en el Grupo "B" han sido encuestados 14 , con ellos no se han usado las herramientas antes mencionadas, las medias obtenidas han sido 52.31 y 36.93 respectivamente. En la Tabla 2 se analiza si las medias son representativas o son fruto de la casualidad.

Tabla 2. Prueba $T$ de Student para muestras independientes.

\begin{tabular}{|c|c|c|c|c|c|c|c|c|c|c|}
\hline & & $\begin{array}{l}\text { Prueb } \\
\text { Leven } \\
\text { la igu } \\
\text { varian }\end{array}$ & $\begin{array}{l}\text { de } \\
\text { para } \\
\text { dad de } \\
\text { as }\end{array}$ & & & Prueba & para la lgu & Idad de Med & & \\
\hline & & & & & & & & & $\begin{array}{r}\text { Inter } \\
\text { confian } \\
\text { la Di }\end{array}$ & $\begin{array}{l}\text { zalo de } \\
\text { ta } 95 \% \text { de } \\
\text { erencia }\end{array}$ \\
\hline & & & & & & & & & Inferior & Superior \\
\hline$\frac{\pi}{\pi}$ & $\begin{array}{l}\text { Se asume } \\
\text { igualdad de } \\
\text { varianzas }\end{array}$ & 9.81 & .004 & 4.15 & 25.00 & .000 & 15.38 & 3.71 & 7.74 & 23.02 \\
\hline$\stackrel{5}{\supset}$ & $\begin{array}{l}\text { Igualdad de } \\
\text { varianzas no } \\
\text { asumidas }\end{array}$ & & & 4.27 & 16.49 & .001 & 15.38 & 3.60 & 7.77 & 22.99 \\
\hline
\end{tabular}

En la Tabla 2 se detecta la prueba $F$ de Fisher identificada como $F$, la intención es detectar diferencias de distribución a nivel estadístico, dentro de la prueba de Levene se prioriza el nivel de significancia, identificado dentro de la Tabla 2 como Sign. Para interpretar dicho dato se debe determinar si es igual o menor a 0.05 , como se observa en la tabla se ha obtenido un valor de 0.004 claramente menor a 0.05 (0.004 $>0.05)$, esto nos permite elegir lgualdad de Varianzas no Asumidas.

Es posible detectar que el uso de herramientas 
síncronas impacta de forma positiva la satisfacción del alumnos, por lo que con los datos mostrados es posible indicar: Al incorporar herramientas síncronas en la comunicación de los alumnos en CUNorte y su sistema semi-presencial, los estudiantes las aceptan y expresan buena satisfacción en el proceso comunicativo, además de sentirse miembros activos de dicho proceso al compararlos con aquellos que no las utilizan. A continuación se presentan los datos recabados a través del cuestionario.

En primera instancia se identificó que los grupos de edades de los alumnos participantes (ambos grupos) se encuentran entre 18 y 26 años. La participación de alumnos varones en relación de las mujeres fue de 6:1. La Ingeniería en Electrónica y Computación es mayormente demandada por hombres, viéndose reflejado en la encuesta que se ha aplicado.

Dentro de los resultados que se obtuvieron en la dimensión: Satisfacción del Alumno en la Comunicación con sus Compañeros y su Profesor, se identifica que los alumnos que utilizaron WhatsApp y Facebook se identifican con escalas mayores de satisfacción cuando se les cuestiona por las Habilidades Interpersonales demostradas por su Profesor, en este sentido, $53.8 \%$ se identifican como Muy Satisfechos, en ningún caso de los alumnos que no utilizaron dichas herramientas se expresan en dicha escala.

Resultados similares se detectan al cuestionar al alumno por la Ayuda recibida del profesor, en el Grupo A se expresan como Muy Satisfechos $61.5 \%$, en el Grupo $B$ se identifica $7.1 \%$ en el mismo nivel de satisfacción. Se interrogó a los alumnos en relación a la Disponibilidad que percibe de su profesor, $100 \%$ de los alumnos del Grupo A se identifican como Satisfechos o Muy Satisfechos, en cambio los alumnos del Grupo $B$ se identifican dichas escalas con un $35.7 \%$.
El resto de los ejes que se plantearon para la dimensión corresponden a: Esfuerzo del profesor al ayudar, Ambiente de confianza que genera el docente, Rapidez con la que se dio respuesta, Ayuda recibida por parte de los compañeros (otros alumnos), Desempeño académico por la ayuda de los compañeros, en todos los casos se detecta que las escalas de satisfacción de los alumnos son mayores cuando se utiliza WhatsApp y Facebook, es decir se ubican a los alumnos entre Satisfechos o Muy Satisfechos con el $100 \%$ de los encuestados, en cambio los alumnos que no utilizan dichas herramientas presentan en promedio $45.7 \%$ en escalas de Indiferencia, Poco Satisfechos o Insatisfechos, ubicando en promedio únicamente al $54.3 \%$ de los estudiantes como Satisfechos o Muy Satisfechos.

Finalmente los hallazgos en los datos que miden la satisfacción del alumno en relación a la comunicación entre sus Compañeros y Profesor (cada una por separado) con el uso de herramientas en línea son presentados en la 
Figura 1. Resultados de los ítems 4 y 12
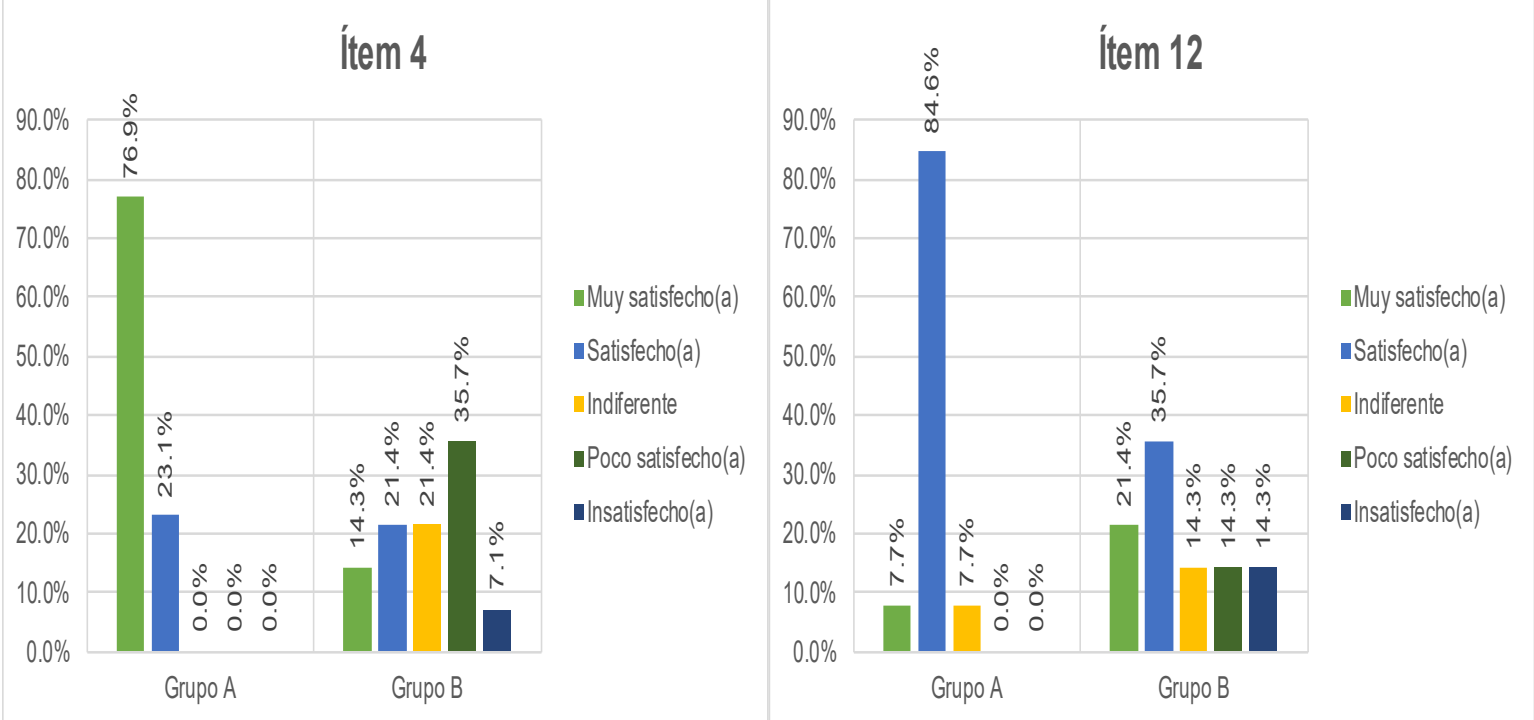

Resulta relevador encontrar que los alumnos del Grupo A siente una mayor satisfacción al interactuar con su profesor que al hacerlo con sus compañeros de clase, el resultado es sorpresivo ya que se esperaban datos similares en ambos ítems.

Si bien la investigación no se centra en los detalles concretos del ¿por qué los alumnos prefieren la comunicación hacia un grupo (profesores) y no de la misma forma con sus compañeros? Aun cuando se da en el mismo nivel de abundancia, nos hemos basado en la experiencia para indicar que el alumno no se

siente del todo cómodo cuando un compañero lo corrige o ayuda, es probable que esto surja de la propia competencia que existe entre ellos, el no demostrar que un similar pueda tener mayores habilidades que él.

Los resultados obtenidos en la dimensión el 246 Alumnocomomiembroactivo de lacomunicación, al usar herramientas en línea, son mostrados en la Tabla 3, en ellos es posible observar que de nueva cuenta el nivel de satisfacción en mayor en el Grupo A con respecto al Grupo B. 
Tabla 3. Resultados en la dimensión el Alumno como miembro activo de la comunicación.

\begin{tabular}{|c|c|c|c|c|c|c|c|c|}
\hline \multirow[b]{3}{*}{ Ítem } & \multicolumn{3}{|c|}{ Grupo A } & \multicolumn{5}{|c|}{ Grupo B } \\
\hline & \multicolumn{3}{|c|}{ Porcentajes } & \multicolumn{5}{|c|}{ Porcentajes } \\
\hline & 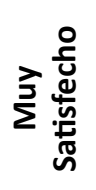 & 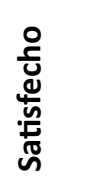 & 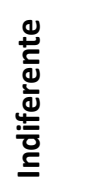 & 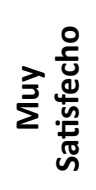 & 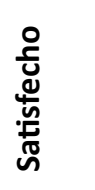 & 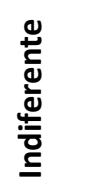 & 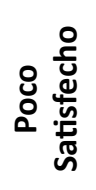 & 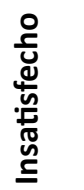 \\
\hline $\begin{array}{l}\text { Ambiente de confianza } \\
\text { con el profesor. }\end{array}$ & 61.5 & 38.5 & 0 & 14.3 & 42.9 & 21.4 & 21.4 & 0 \\
\hline $\begin{array}{l}\text { Ambiente de confianza } \\
\text { con sus compañeros. }\end{array}$ & 30.8 & 69.2 & 0 & 14.3 & 42.9 & 21.4 & 21.4 & 0 \\
\hline $\begin{array}{l}\text { El alumno ayuda a sus } \\
\text { compañeros. }\end{array}$ & 46.2 & 53.8 & 0 & 21.4 & 57.1 & 14.3 & 0 & 7.1 \\
\hline $\begin{array}{l}\text { Profesor y alumnos } \\
\text { ayudan al estudiante. }\end{array}$ & 46.2 & 46.2 & 7.7 & 35.7 & 28.6 & 21.4 & 7.1 & 7.1 \\
\hline $\begin{array}{l}\text { Los compañeros } \\
\text { resolvían dudas del } \\
\text { alumno. }\end{array}$ & 53.8 & 46.2 & 0 & 28.6 & 35.7 & 21.4 & 7.1 & 7.1 \\
\hline $\begin{array}{l}\text { Saber que el profesor } \\
\text { podía resolver dudas } \\
\text { del alumno }\end{array}$ & 53.8 & 46.2 & 0 & 35.7 & 35.7 & 7.1 & 7.1 & 14.3 \\
\hline $\begin{array}{l}\text { Lenguaje usado entre } \\
\text { alumno y profesor. }\end{array}$ & 38.5 & 53.8 & 7.7 & 21.4 & 28.6 & 21.4 & 14.3 & 14.3 \\
\hline $\begin{array}{l}\text { Ambiente activo de } \\
\text { participación }\end{array}$ & 53.8 & 46.2 & 0 & 42.9 & 28.6 & 14.3 & 0 & 14.3 \\
\hline $\begin{array}{l}\text { Ayuda desde la } \\
\text { comunicación en línea }\end{array}$ & 38.5 & 61.5 & 0 & 35.7 & 28.6 & 14.3 & 7.1 & 14.3 \\
\hline
\end{tabular}

Nota: aEn el Grupo A las escalas Poco Satisfecho e Insatisfecho han sido omitidas ya que no fueron expresadas por los encuestados. ${ }^{b} \mathrm{En}$ todos los casos los datos representan porcentajes.

Dentro de la investigación se cuestionó a los alumnos que han usado WhatsApp y Facebook su satisfacción. Para cada una de las herramientas se generó una interrogante similar, por ejemplo: La incorporación de WhatsApp como herramienta de comunicación en clases, ¿me hace sentir? y La incorporación de Facebook como herramienta de comunicación en clases, ¿me hace sentir? Los datos son mostrados en la Tabla 4: 
Tabla 4. Satisfacción de los alumnos con respecto a WhatsApp y Facebook.

\begin{tabular}{|c|c|c|c|c|c|c|}
\hline \multirow[b]{2}{*}{ Ítem } & \multicolumn{3}{|c|}{ WhatsApp } & \multicolumn{3}{|c|}{ Facebook } \\
\hline & 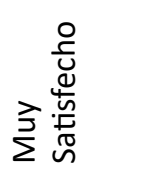 & 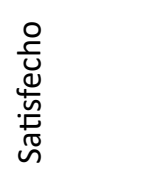 & 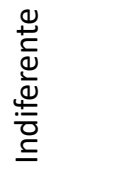 & 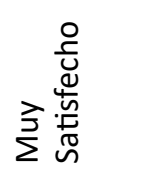 & 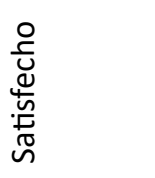 & 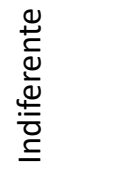 \\
\hline $\begin{array}{l}\text { Su incorporación como } \\
\text { herramienta de comunicación. }\end{array}$ & $69.2 \%$ & $30.8 \%$ & $0 \%$ & $38.5 \%$ & $38.5 \%$ & $23.0 \%$ \\
\hline Rapidez al compartir archivos. & $61.5 \%$ & $30.8 \%$ & $7.7 \%$ & $53.8 \%$ & $30.8 \%$ & $15.4 \%$ \\
\hline $\begin{array}{l}\text { Logros, ayudas rápidas, dudas } \\
\text { solventadas. }\end{array}$ & $46.2 \%$ & $53.8 \%$ & $0 \%$ & $30.8 \%$ & $53.8 \%$ & $15.4 \%$ \\
\hline
\end{tabular}

WhatsApp se posicionó como herramienta favorita sobre Facebook, lo pudimos observar en la Tabla 4, además de ello se generó la interrogante en la que se cuestiona al alumno si recomienda el uso de cada una de ellas en otros cursos/clases, los resultados son observados en la Figura 2.

Figura 2. Se recomienda su uso en otros cursos.

Se realizó un análisis de la forma y el número de veces que los alumnos y el profesor utilizaron WhatsApp y Facebook, los resultados son presentados en la Tabla 5.

\begin{tabular}{rrrr} 
Tabla $5 . \quad$ Grupo $\quad$ "A": $\quad$ Uso de & WhatsApp & Facebook \\
\hline Actividad realizada & WhatsApp & Facebook \\
\hline Conversaciones iniciadas por el profesor: & 437 & 147 \\
Conversaciones iniciadas por los alumnos: & 1309 & 142 \\
Documentos compartidos por el profesor: & 21 & 0 \\
Documentos compartidos por los alumno: & 15 & 0 \\
Archivos compartidos por el profesor (fotos, stickers y gifs): & 87 & 11 \\
Archivos compartidos por los alumnos (fotos, stickers y gifs): & 230 & 19 \\
Mensajes de audio compartidos por el profesor: & 4 & 0 \\
Mensajes de audio compartidos por los alumnos: & 8 & 0 \\
Videos compartidos por el profesor: & 5 & 0 \\
Videos compartidos por los alumnos: & 1 & 0 \\
\hline Total: & $\mathbf{2 1 1 7}$ & $\mathbf{3 1 9}$ \\
\hline
\end{tabular}


La Tabla 5 nos muestra la forma copiosa y variada en la que los alumnos utilizan WhatsApp en comparación con Facebook, ya que la diferencia de uso representa $86.9 \%$ y $13.1 \%$ respectivamente (al revisar la suma de los totales de uso en cada herramienta), como investigadores se ha tomado como indicativo la necesidad de ofrecer más de una herramienta, para que el alumno elija con la que se sienta cómodo y no se trate de una imposición a la que se tenga que apegar.

\section{CONCLUSIONES}

Después de realizar la investigación nos sentimos con mayor confianza de sugerir la utilización de herramientas con las que el alumno se identifica y que utiliza en su vida diaria, es más sencillo acercar al alumno a través de medios que utiliza cotidianamente al aprendizaje y la comunicación que hacerlo desde estrategias que se han dictado desde experiencias ajenas al propio alumno.

Si bien el primer paso que se ha dado en la investigación no concluye hasta este punto ya que es prudente volver a replicarlo con una cantidad más representativa de alumnos y considerando que el primer análisis fue realizado hasta antes de que a nivel mundial nos viéramos envueltos en una pandemia que ha venido a replantear todas las actividades que cotidianamente se hacían, entre ellas la educación y comunicación con los alumnos, es prudente plantear un análisis futuro donde se considere que instituciones como CUNorte se han visto en la necesidad de replantear la comunicación con sus alumnos y WhatsApp ha tomado un papel muy importante en esa realidad a la que se nos ha orillado, ante un panorama así resulta prudente interrogar al estudiante en sus niveles de satisfacción, y claro, la satisfacción comunicativa se vuelve eje fundamental en esos análisis.

Es necesario plantear la satisfacción del alumno como eje primordial en su educación y preparación, si bien la investigación se ha centrado en el quehacer comunicativo, resulta trascendental realizar análisis constantes de las estrategias que se sigue para acercar al alumno con la preparación, entendemos que si logramos alumnos satisfechos en consecuencia este será capaz de apropiarse de los conocimientos que la sociedad demanda de los nuevos profesionistas en la que ella invierte como punto de crecimiento generalizado.

Únicamente nos resta indicar que es necesario que los investigadores estemos atentos en recabar la información que nos de indicios de la prudencia de plantear o potencializar estrategias en la educación, es posible que en dicho análisis encontremos que algunos procesos están en decadencia y otros emergen, nuestra responsabilidad será mostrar dichos descubrimientos a nuestras comunidades educativas y por lo tanto a la sociedad en la que nos desenvolvemos.

\section{REFERENCIAS BIBLIOGRÁFICAS}

Alfaro, A. P., Fernández, M. S. y Alvarado, R. I. (2014). El uso de las TIC en la formación permanente del profesorado para la mejora de su práctica docente. Revista científica electrónica de Educación y Comunicación en la Sociedad del Conocimiento, 1(14), 7095. https://dialnet.unirioja.es/servlet/ articulo?codigo $=5226664$.

Alonso, M., Gonzálvez, J. y Muñoz, A. (2016). Ventajas e inconvenientes del uso de dispositivos electrónicos en el aula: percepción de los estudiantes de grados en comunicación. Revista de Comunicación de la SEECI, 20(41), 136-154.

Álvarez, J., Chaparro, E. y Reyes, D. (2015). Estudio de la satisfacción de los estudiantes con los servicios educativos brindados por instituciones de educación 
superior del Valle de Toluca. REICE. Revista Iberoamericana sobre Calidad, Eficacia y Cambio en Educación, 13(2).

Amayuela, G. (2017). Comunicación y su relación con la educación en el contexto universitario. Alternativas en Psicología, 1, 8-19.

Ardura, D. y Zamora, A. (2013). ¿Son útiles entornos virtuales de aprendizaje en la enseñanza de las ciencias secundaria? Evaluación de una experiencia en la enseñanza y el aprendizaje de la Relatividad. Revista Eureka sobre Enseñanza y Divulgación de las Ciencias, 11(1), 83-93.

Arteaga, L., Ayala, C., Castillo, E., Seclén, L., Merino, J., Simón, D., Trujillo, A., Shimizu, R., Zúñiga, J., Vallejo, G., Zambrano, J., Julca, J., Samamé, F., Mattos, N., Aldave, K., Charcape, H. y Meléndez, A. (2018). Influencia de la altura en los niveles de proteína $C$ reactiva en adultos con adiposidad visceral abdominal. Horizonte Médico (Lima), 18(1), 29-34. https://doi. org/10.24265/horizmed.2018.v18n1.05

Camacho, C. y Becerra, G. M. (2016). El rol articulador del profesor: una perspectiva social y tecnológica. Paakat: Revista de Tecnología y Sociedad, (10), [fecha de Consulta 24 de Agosto de 2020]. ISSN: Disponible en: https://www.redalyc.org/ articulo.oa?id=4990/499054322005.

Castro, M. y Jiménez, L. (2008). Caso Unionconsulting: estrategias para el mejoramiento de la comunicación interna Universidad Javeriana. Colombia: Pontificia Universidad Javeriana.

Cisneros, L. y Robles, S. (2017). ¿Para qué utilizan el celular en el aula, los estudiantes universitarios? Consejo
Mexicano de Investigación Educativa A.C. 1-17.

Cook, D. (2013). ¿Debo utilizar el aprendizaje en línea? Investigación en Educación Médica, 2 (5), 3-6.

Cortez, K., Fuentes, V., Villablanca, I. y Guzmán, C. (2013). Creencias docentes de profesores ejemplares y su incidencia en las prácticas pedagógicas. Estudios Pedagógico, 39(2), 97-113.

Escobar, M. (2015). Influencia de la interacción alumno-docente en el proceso enseñanza-aprendizaje. Paakat: Revista de Tecnología y Sociedad, (8).

Escofet, A., Folgueiras, P., Luna, E. y Palou, B. (2016). Elaboración y validación de un cuestionario para la valoración de proyectos de aprendizaje-servicio. Revista Mexicana de Investigación Educativa, 21(70), 929-949.

Fernández, I., Riveros, V. y Montiel, G. (2017). Software educativo y las funciones matemáticas. Una estrategia de apropiación. Omnia, 23 (1), 9-19.

Free Software Foundation, Inc. (2020, mayo 8). PSPP - GNU Project - Free Software Foundation. Recuperado 2 de junio de 2020, de https://www.gnu.org/software/ pspp/get.html

George, C. y Trujillo, L. (2018). Aplicación del Método Delphi Modificado para la validación de un cuestionario de incorporación de las TIC en la práctica docente. Revista Iberoamericana de Evaluación Educativa, 11(1), 113-135. https://doi.org/10.15366/ riee2018.11.1.007

Giasanti, A. R. P., Taboada, A. P. y Jansiski, L. (2016). Uso de la aplicación WhatsApp 
por estudiantes de Odontología de Sao Paulo, Brasil. Revista Cubana de Información en Ciencias de la Salud, 27(4), 503-514. http://scielo.sld.cu/ scielo.php?script=sci_arttext\&pid $=$ S2307-21132016000400007

Gómez, M., Washington, J. y Rivera, A. (2017). Comunicación docente, un desafío epistemológico para el autoaprendizaje. EduSol, 17 (60), 60-68.

González, F., Escoto, M. C., y Chávez, J. K. (2017). Estadística aplicada en Psicología y Ciencias de la Salud. Ciudad de México, México: Manual Moderno.

Hernández, E. (2015). Factores determinantes en la comunicación de los procesos de innovación docente. Aplicación en los Centros de Enseñanza Secundaria Públicos de la Región de Murcia. Murcia, España: Universidad de Murcia.

Hernández, R. M. (2017). Impacto de las TIC en la educación: Retos y Perspectivas. Propósitos y Representaciones, 5(1), 325-347. https://doi.org/10.20511/ pyr2017.v5n1.149

Herrera, K., Acuña, M. y Gil, L. (2017). Motivación de jóvenes universitarios hacia el uso de teléfonos celulares. Revista Encuentros, Universidad Autónoma del Caribe, 15(1), 91-105. https://doi.org/10.15665/ re.v15i1.631.

Hershkovizt, A. y Forkosh-Baruch, A. (2017). La relación profesor-alumno y la comunicación en Facebook: percepciones de los alumnos. Comunicar, XXV (53), 91-101.

López, P. y Fachelli, S. (2015). Metodología de la investigación social cuantitativa. Barcelona, España: Universitat
Autònoma de Barcelona. Recuperado de https://ddd.uab.cat/pub/ caplli/2016/163567/metinvsoccua_ a2016_cap2-3.pdf

Marrero, O. (2016). Comunicación educativa: esencia del aprendizaje en el contexto actual de la educación superior. In R. Mancinas (Ed.), Actas del I Congreso Internacional Comunicación y Pensamiento: comunicracia y desarrollo social (pp. 1420-1427). Sevilla, España: Egregius.

Martínez, JR., Carralero, Y., Falcón, Y., Guevara, R. y Peña, ID. (2016). Influencia de los estilos de aprendizaje en el rendimiento académico en Morfofisiología. Revista Electrónica Dr. Zoilo E. Marinello Vidaurreta. 41(7). Disponible en: http:// revzoilomarinello.sld.cu/index.php/zmv/ article/view/709.

Medina, R., Medina de la Rosa, R. y Moreno, M. (2017). Pensamiento crítico y aprendizaje grupal: vía para mejorar la comunicación en alumnos universitarios. Revista Universidad y Sociedad, 9(4), 168-176. Recuperado en 08 de febrero de 2019, de http://scielo.sld.cu/scielo. php?script=sci_arttext $\&$ pid $=$ S2218$36202017000400023 \&$ Ing=es\&tlng=es.

Mora, F. (2014). Gestión de una comunicación escrita eficaz del tutor virtual durante los procesos de enseñanza aprendizaje en los cursos en línea en la UNED. INNOVACIONES EDUCATIVAS, 15(20), 23-36.

Morales, J., Montes, R., Zermeño, N., Duran, J., y Herrera, F. (2018). The use of Fuzzy Linguistic Information and Fuzzy Delphi Method to validate by consensus a questionnaire in a Blended-Learning Environment. Communications in 
Computer and Information Science, 855.

Otzen, T. y Manterola, C. (2017). Técnicas de muestreo sobre una población a estudio. International Journal of Morphology, 35(1), 227-232.

Padilla, J. (2008). Factores de importancia de la educación virtual. Grupo Alfa, 1-17.

Pérez, F., Martínez, P. y Martínez, M. (2015). Satisfacción del estudiante universitario con la tutoría. Diseño y validación de un instrumento de medida. ESTUDIOS SOBRE EDUCACIÓN, 29, 81-101. https://doi.org/10.15581/004.29.81-101.

Pérez, I. y Pereyra, E. (2015). Satisfacción estudiantil: un indicador de la calidad educativa en el departamento de biología celular, UCV. Revista de Pedagogía, 36(99), 69-89.

Ramírez, J. e Hidalgo, F. (2018). Satisfacción de estudiantes de secundaria nocturna y su incidencia en el abandono escolar. Revista Electrónica Educare. 22 (1), 287-300. http://dx.doi.org/10.15359/ ree.22-1.14.

Ruiz, J. Bernardez, R. y Martínez, A. (2016). Educación formal e informal mediada por tecnologías. Las propuestas de formación abierta y masiva. MOHOS, REA, PLE. 2015 IX Conferencia Internacional Guide, 1-13.

Santoveña, C. S. M. (2012). El proceso de enseñanza-aprendizaje a través de herramientas de comunicación síncrona: El caso de Elluminate Live. Electronic Journal of Research in Educational Psychology, 10 (1), 447-474.

Sanz, J. (2014). WhatsApp: potencialidad educativa versus dependencia y adicción. Revista DIM, (30), 1-20.
Recuperado de http://dimglobal.net/ revistaDIM30/revista30bisOCwhatsapp. $\mathrm{htm}$

Secretaria de Educación Pública. (1993, 13 julio). Ley General de Educación. https:// www.sep.gob.mx/work/models/sep1/ Resource/558c2c24-0b12-4676-ad908ab78086b184/ley_general_educacion. pdf

Sepúlveda, M. (2016). La relación educación comunicación desde la virtualidad, una perspectiva. Revista de la Facultad de Estudios en Ambientes Virtuales, 35-59.

Solana. F (2011). ¿Qué significa calidad en la educación? México: Noriega Editores.

Surdez, E., Sandoval, M. y Lamoyi, C. (2018). Satisfacción estudiantil en la valoración de la calidad educativa universitaria. Educación y Educadores, 21 (1), 9-26. http://dx.doi.org/10.5294/ edu.2018.21.1.1.

Tuapanta, J. V., Duque, M. A. y Mena, A. P. (2017). Alfa de Cronbach para validar un cuestionario de uso de TIC en docentes universitarios. mktDescubre, 10(1), 3748.

Turpo, O. (2013). Perspectiva de la convergencia pedagógica y tecnológica en la modalidad Blended Learning. REDRevista de Educación a Distancia, (39), $1-14$.

Vaca, B., Cela, J. y Gallardo, E. (2016). La comunicación en entornos simulados para el aprendizaje. Revista Iberoamericana de Educación, 72(2), 85-102.

Viñals, A. y Cuenca, J. (2016). El rol del docente en la era digital. Revista Interuniversitaria de Formación del Profesorado, 30 (2), 103-114. 